\title{
Types, terms and reductions:
}

\section{A special issue dedicated to Paweł Urzyczyn for his 65th birthday}

\section{Preface}

It is our honour to give this volume to the readers, which we wish to dedicate to Paweł Urzyczyn for his 65 th birthday. His spirit of engagement in solving of puzzling riddles posed by logical phenomena and love of mathematical fine detail led throughout the years to many valuable achievements and opened new paths that are remarkably attractive to follow. Here is a short account of his work, with emphasis on the subjects pursued in this volume.

In his early years of scientific activity Paweł worked on formal structures that describe behaviour of imperative programs and make it possible to conduct formal reasoning on their correctness such as dynamic logic or Hoare logic. It may come as a surprise for people form the $\lambda$ calculi community, that in one of the papers from these years he defined a Hoare logic for arrays, which in today's terminology should rather be called dictionaries.

However, he switched his interests soon after that work to lambda calculi with types. Many of his results in the 1990's concerned the undecidability and complexity of various problems related to type reconstruction and subtyping for different type systems considered in direct connection with functional programming, that is ML, simple types, System F, and also beyond that, i.e. System $\mathrm{F}_{\omega}$ and intersection type systems. These results were accompanied by studies on type inhabitation problems, which can be formulated in a very close way to type reconstruction problems. These investigations of algorithmic properties were sometimes diversified by studies related to semantics of logical systems, which can be observed in his papers on parallel observers or cut-elimination.

One of the most important threads in the work of Paweł were the studies on reconstruction problems in intersection type discipline. His contributions in this field mapped very densely the knowledge concerning the complexity and undecidability for these systems.

Due to the growing interest in dependently typed programming languages and proof systems based on intuitionistic calculi he turned his activity to study the undecidability and complexity of type inhabitation (or provability) in the most basic dependent types formalism of first-order minimal logic, a system in which classical or even intuitionistic or coherent logic are just theories. 
The expertise of Paweł made him an invaluable partner in discussions concerning many topics ranging from programming language design (we can mention here discussions on Magda programming language), through design of program synthesis algorithms (cf. his contributions to bounded combinatory logic) to provability in first-order logic (cf. his contributions from the last years).

The idea to celebrate Pawel's birthday and commemorate his scientific achievements with a volume met enthusiastic support of the chief editor of Fundamenta Informaticae, Damian Niwiński. We would like to thank him here for making this undertaking possible and for his continuous care over the whole process. We sent out our invitation to various scientific centres where the topics Paweł was interested in were studied over the years of his career, form Japan to USA. We wish to thank all authors who have submitted their contributions. As this volume had to be prepared within certain time limits, we could not afford unlimited time for improvement and we had to decide to reject, with our regret, some contributions that would be valuable but were not ready in time.

All the papers we had received were peer reviewed according to the high standard of Fundamenta Informaticae. In addition we included in the volume one personal account of relations between computer science and logic written by Assaf Kfoury, one of the Paweł's nearest scientific collaborators. In this way, a broader perspective of the studies Paweł took part in over years is brought closer to the readers.

Editors of this special issue

Thorsten Altenkirch University of Nottingham

Aleksy Schubert University of Warsaw 